\title{
Evolution of the universe with flat extra dimensions
}

\author{
Je-An Gu, W.-Y.P. Hwang, Jr-Wei Tsai \\ Department of Physics, National Taiwan University, Taipei 106, Taiwan
}

Received 23 April 2004; received in revised form 2 July 2004; accepted 5 August 2004

Available online 7 September 2004

\begin{abstract}
Evolution of a universe with homogeneous extra dimensions is studied with the benefit of a wellchosen parameter space that provides a systematic, useful, and convenient way for analysis. In this model we find a natural evolution pattern that entails not only stable extra dimensions in the radiationdominated era, thereby preserving essential predictions in the standard cosmology, but also the present accelerating expansion while satisfying the limit on the variation of Newtonian gravitational constant. In this natural evolution pattern the extra dimensions tend to be stabilized automatically without resorting to artificial mechanisms in both the radiation-dominated and the matter-dominated era, as a wonderful feature for building models with extra dimensions. In addition, the naturalness of this evolution pattern that guarantees the late-time accelerating expansion of a matter-dominated universe presents a solution to the coincidence problem: why the accelerating phase starts at the present epoch. The feasibility of this evolution pattern for describing our universe is discussed.
\end{abstract}

(c) 2004 Elsevier B.V. All rights reserved.

PACS: 98.80.Es; 04.50.+h; 98.80.Jk

\section{Introduction}

The observations by Supernova Cosmology Project and Supernova Search Team have suggested in 1998 that the expansion of the present universe is accelerating [1,2]. This conclusion is reinforced recently in 2003 by WMAP measurements [3]. One general conclusion from these measurements and the $\mathrm{CMB}$ observations in recent years [3-5] is that

E-mail addresses: jagu@phys.ntu.edu.tw (J.-A. Gu), wyhwang@phys.ntu.edu.tw (W.-Y.P. Hwang). 
the universe has the critical density, consisting of $1 / 3$ of ordinary matter and $2 / 3$ of dark energy with a negative pressure [6] (such that $p_{x} / \rho_{x}<-0.78$ [3]). Although this acceleration may be driven by the existence of a positive cosmological constant (vacuum energy) [7], there remain other interpretations of the accelerating expansion, such as "quintessence" (a slowly evolving scalar field) [8,9] and the presence of extra dimensions [10,11].

The presence of extra dimensions is required in various theories beyond the standard model of particle physics, especially in the theories for unifying gravity and other forces, such as superstring theory. Extra dimensions should be "hidden" (or "dark") for consistency with observations. Various scenarios for "hidden" extra dimensions have been proposed, for example, a brane world with large compact extra dimensions in factorizable geometry proposed by Arkani-Hamed et al. [12] (see also [13]), and a brane world with noncompact extra dimensions in nonfactorizable geometry proposed by Randall and Sundrum [14]. In this paper we will employ the simplest scenario: small compact extra dimensions in factorizable geometry, as introduced in the Kaluza-Klein (KK) theories [15].

The possibility of generating the accelerating expansion of the present matter-dominated universe via the evolution of homogeneous and isotropic extra dimensions is first pointed out by $\mathrm{Gu}$ and Hwang [10] (for the late-time accelerating expansion in the brane-world scenarios, see [16-19]). Furthermore, the general idea of unifying dark energy sources (i.e., dark matter and dark energy) and dark geometry (e.g., extra dimensions) into one has been sketched by Gu [11], who pointed out that dark geometry (instead of dark energy) can be an intriguing candidate for generating accelerating expansion. As indicated by Einstein's general relativity, mass (energy) and geometry are two faces of one single nature, therefore it is biased to consider only mass (energy) has dark part, while believing blindly that all geometry is totally "visible" to our poor eyes [11].

In this simple scenario making use of a highly symmetric extra space, there are much fewer free parameters (only two additional degrees of freedom, the expansion rate and the curvature of the extra space, in addition to those in the standard cosmological models without dark energy) so that it is much easier to be ruled out, compared with the quintessence models, by constraints from observations. In particular, an essential difficulty of this model [20] stems from the constraint on the variation of the Newtonian gravitational constant, which will be produced along with the evolution of extra dimensions that is the key element for generating accelerating expansion.

In this paper we will study a more general case in which the evolution of our universe in various eras, especially the present accelerating expansion era, is governed not only by the matter contents (excluding dark energy) therein, but also by the curvature of the ordinary 3 -space and the evolution of extra dimensions. We will explore the feasibility of this model for generating accelerating expansion while satisfying observational constraints, especially the variation of the Newtonian gravitational constant. Through these studies we will also present several nice features of this model, such as the automatic stabilization of extra dimensions and the solution to the cosmic coincidence problem-why the energy densities of dark energy and dark matter are comparable now (i.e., "why now" problem), or, more precisely (if dark energy is not a necessary ingredient for the accelerating expansion), why the accelerating phase of our universe starts at the present epoch. 


\section{The higher-dimensional world: basics}

We consider a $(3+n+1)$-dimensional space-time where $n$ is the number of extra spatial dimensions. Assuming that both the three-dimensional ordinary space and the $n$ dimensional extra space are homogeneous and isotropic, we use two spatial parts of the Robertson-Walker metric to describe this space-time as follows:

$$
d s^{2}=d t^{2}-a^{2}(t)\left(\frac{d r_{a}^{2}}{1-k_{a} r_{a}^{2}}+r_{a}^{2} d \Omega_{a}^{2}\right)-b^{2}(t)\left(\frac{d r_{b}^{2}}{1-k_{b} r_{b}^{2}}+r_{b}^{2} d \Omega_{b}^{2}\right),
$$

where $a(t)$ and $b(t)$ are scale factors, and $k_{a}$ and $k_{b}$ relate to curvatures of the ordinary 3space and the extra space, respectively. The value of $r_{b}$ is set to be within the interval $[0,1)$ corresponding to the finite size of extra dimensions. Assuming that the matter content in this higher-dimensional space is a perfect fluid with the energy-momentum tensor

$$
T_{\beta}^{\alpha}=\operatorname{diag}\left(\bar{\rho},-\bar{p}_{a}, \ldots,-\bar{p}_{b}, \ldots\right),
$$

we can write the Einstein equations $[10,11]$ as

$$
\begin{aligned}
& 3\left[\left(\frac{\dot{a}}{a}\right)^{2}+\frac{k_{a}}{a^{2}}\right]+\frac{n(n-1)}{2}\left[\left(\frac{\dot{b}}{b}\right)^{2}+\frac{k_{b}}{b^{2}}\right]+3 n \frac{\dot{a}}{a} \frac{\dot{b}}{b}=8 \pi \bar{G} \bar{\rho}, \\
& 2 \frac{\ddot{a}}{a}+n \frac{\ddot{b}}{b}+\left[\left(\frac{\dot{a}}{a}\right)^{2}+\frac{k_{a}}{a^{2}}\right]+\frac{n(n-1)}{2}\left[\left(\frac{\dot{b}}{b}\right)^{2}+\frac{k_{b}}{b^{2}}\right]+2 n \frac{\dot{a}}{a} \frac{\dot{b}}{b}=-8 \pi \bar{G} \bar{p}_{a}, \\
& 3 \frac{\ddot{a}}{a}+(n-1) \frac{\ddot{b}}{b}+3\left[\left(\frac{\dot{a}}{a}\right)^{2}+\frac{k_{a}}{a^{2}}\right]+\frac{(n-1)(n-2)}{2}\left[\left(\frac{\dot{b}}{b}\right)^{2}+\frac{k_{b}}{b^{2}}\right] \\
& +3(n-1) \frac{\dot{a}}{a} \frac{\dot{b}}{b}=-8 \pi \bar{G} \bar{p}_{b},
\end{aligned}
$$

where $\bar{\rho}$ is the energy density in the higher-dimensional world, and $\bar{p}_{a}$ and $\bar{p}_{b}$ are the pressures in the ordinary 3 -space and the extra space, respectively.

The terms involving the scale factor $b$ in Eqs. (3) and (4) are additional terms and Eq. (5) is an additional equation coming from extra dimensions. As pointed out by $\mathrm{Gu}$ in [11], if extra dimensions exist but we are so biased that we admit only mass/energy can have dark part while blindly believing there is no dark geometry (such as extra dimensions) at all, these additional terms from extra dimensions will automatically be moved from the lefthand side of the Einstein equations (describing geometry) to the right-hand side (describing energy contents) and be treated as some sort of effective dark energy sources, as indicated in the following equations (from rearranging Eqs. (3)-(5)):

$$
\begin{aligned}
& 3\left[\left(\frac{\dot{a}}{a}\right)^{2}+\frac{k_{a}}{a^{2}}\right]=8 \pi \bar{G}\left(\bar{\rho}+\bar{\rho}_{\mathrm{eff}}\right), \\
& 2 \frac{\ddot{a}}{a}+\left[\left(\frac{\dot{a}}{a}\right)^{2}+\frac{k_{a}}{a^{2}}\right]=-8 \pi \bar{G}\left(\bar{p}_{a}+\bar{p}_{a, \text { eff }}\right), \\
& 3 \frac{\ddot{a}}{a}+3\left[\left(\frac{\dot{a}}{a}\right)^{2}+\frac{k_{a}}{a^{2}}\right]=-8 \pi \bar{G}\left(\bar{p}_{b}+\bar{p}_{b, \mathrm{eff}}\right),
\end{aligned}
$$


where

$$
\begin{aligned}
& 8 \pi \bar{G} \bar{\rho}_{\mathrm{eff}} \equiv-\frac{n(n-1)}{2}\left[\left(\frac{\dot{b}}{b}\right)^{2}+\frac{k_{b}}{b^{2}}\right]-3 n \frac{\dot{a}}{a} \frac{\dot{b}}{b}, \\
& 8 \pi \bar{G} \bar{p}_{a, \text { eff }} \equiv n \frac{\ddot{b}}{b}+\frac{n(n-1)}{2}\left[\left(\frac{\dot{b}}{b}\right)^{2}+\frac{k_{b}}{b^{2}}\right]+2 n \frac{\dot{a}}{a} \frac{\dot{b}}{b}, \\
& 8 \pi \bar{G} \bar{p}_{b, \text { eff }} \equiv(n-1) \frac{\ddot{b}}{b}+\frac{(n-1)(n-2)}{2}\left[\left(\frac{\dot{b}}{b}\right)^{2}+\frac{k_{b}}{b^{2}}\right]+3(n-1) \frac{\dot{a}}{a} \frac{\dot{b}}{b} .
\end{aligned}
$$

Note that an equation such as Eq. (5) or Eq. (8) is absent in the standard cosmology, that is, a no-dark-geometry believer living in a $(3+1)$-dimensional universe will never notice this equation, while a higher-dimensional cosmologist will realize it as a constraint equation for the behavior of the scale factor $b$ and the pressure in the extra space $\bar{p}_{b}$. In addition, we note that the effects of extra dimensions on the evolution of the universe come from two ingredients: one is the size of extra dimensions, through the (spatial) curvature (i.e., $k_{b} / b^{2}$ ); the other is the evolution of extra dimensions, through the expansion rate $\dot{b} / b$.

Using equations of state $\bar{p}_{a}=w_{a} \bar{\rho}$ and $\bar{p}_{b}=w_{b} \bar{\rho}$, we rearrange Eqs. (4) and (5) and obtain

$$
\begin{aligned}
\frac{\ddot{a}}{a}= & -\left[\frac{(2 n+1)-3(n-1) w_{a}+3 n w_{b}}{n+2}\right]\left(\frac{\dot{a}^{2}}{a^{2}}+\frac{k_{a}}{a^{2}}\right) \\
& +\frac{n(n-1)}{2}\left[\frac{1+(n-1) w_{a}-n w_{b}}{n+2}\right]\left(\frac{\dot{b}^{2}}{b^{2}}+\frac{k_{b}}{b^{2}}\right) \\
& -n\left[\frac{(n-1)-3(n-1) w_{a}+3 n w_{b}}{n+2}\right]\left(\frac{\dot{a} \dot{b}}{a b}\right), \\
\frac{\ddot{b}}{b}= & 3\left(\frac{1-3 w_{a}+2 w_{b}}{n+2}\right)\left(\frac{\dot{a}^{2}}{a^{2}}+\frac{k_{a}}{a^{2}}\right) \\
& -\frac{n-1\left[\frac{(n+4)+3 n w_{a}-2 n w_{b}}{n+2}\right]\left(\frac{\dot{b}^{2}}{b^{2}}+\frac{k_{b}}{b^{2}}\right)}{}-3\left(\frac{2+3 n w_{a}-2 n w_{b}}{n+2}\right)\left(\frac{\dot{a} \dot{b}}{a b}\right) .
\end{aligned}
$$

Before performing detailed numerical analyses, we can extract essential features of the above equations and possible evolution patterns governed by them via new variables

$$
\begin{aligned}
& u(t) \equiv \dot{a} / a, \\
& v(t) \equiv \dot{b} / b,
\end{aligned}
$$

and the parameter space expanded by

$$
\begin{aligned}
& X_{a} \equiv \frac{k_{a}}{a^{2} u^{2}}, \\
& X_{b} \equiv \frac{k_{b}}{b^{2} u^{2}}, \\
& Y \equiv v / u .
\end{aligned}
$$


The use of the parameter space for analyzing the evolution of the universe with extra dimensions was first suggested by $\mathrm{Gu}$ and Hwang in $[10,11]$. In terms of the above new variables, Eqs. (3), (12), and (13) can be rewritten as follows,

$$
\begin{aligned}
\left(1+X_{a}\right)+ & \frac{n(n-1)}{6}\left(Y^{2}+X_{b}\right)+n Y=\frac{8 \pi \bar{G} \bar{\rho}}{3 u^{2}}, \\
\frac{1}{u^{2}}\left(\frac{\ddot{a}}{a}\right)= & \frac{\dot{u}}{u^{2}}+1 \\
= & -\left[\frac{(2 n+1)-3(n-1) w_{a}+3 n w_{b}}{n+2}\right]\left(1+X_{a}\right) \\
& +\frac{n(n-1)}{2}\left[\frac{1+(n-1) w_{a}-n w_{b}}{n+2}\right]\left(Y^{2}+X_{b}\right) \\
& -n\left[\frac{(n-1)-3(n-1) w_{a}+3 n w_{b}}{n+2}\right] Y, \\
\frac{1}{u^{2}}\left(\frac{\dot{b}}{b}\right)= & \frac{\dot{v}}{u^{2}}+Y^{2} \\
= & 3\left(\frac{1-3 w_{a}+2 w_{b}}{n+2}\right)\left(1+X_{a}\right) \\
& -\frac{n-1}{2}\left[\frac{(n+4)+3 n w_{a}-2 n w_{b}}{n+2}\right]\left(Y^{2}+X_{b}\right) \\
& -3\left(\frac{2+3 n w_{a}-2 n w_{b}}{n+2}\right) Y .
\end{aligned}
$$

The above three equations indicate that the essential quantities $\bar{G} \bar{\rho}, \ddot{a} / a$ and $\ddot{b} / b$, in unit of $u^{2}$, are all functions of $X_{a}, X_{b}$ and $Y$ for given values of $n, w_{a}$ and $w_{b}$. That is, $X_{a}, X_{b}$ and $Y$ form a set of variables that can specify the state of a universe with extra dimensions, provided that the unit of time is set to be the Hubble time $u^{-1}$ at each moment. With the use of the parameter space $\left(X_{a}, X_{b}, Y\right)$ we can plot regions for $\bar{\rho}>0, \bar{\rho}=0$, or $\bar{\rho}<0$, and regions for $\ddot{a}>0, \ddot{a}=0$, or $\ddot{a}<0$, in order to catch a basic picture of the state of the universe at each position in the parameter space. In addition, drawing flow vectors in the parameter space is useful for sketching possible evolution patterns. Flow vectors in the parameter space are vector fields:

$$
\frac{d\left(X_{a}, X_{b}, Y\right)}{d t}=u\left[-2 X_{a}\left(1+\frac{\dot{u}}{u^{2}}\right),-2 X_{b}\left(Y+\frac{\dot{u}}{u^{2}}\right),\left(\frac{\dot{v}}{u^{2}}-Y \frac{\dot{u}}{u^{2}}\right)\right],
$$

which, in unit of $u$, are also functions of $\left(X_{a}, X_{b}, Y\right)$ for given values of $\left(n, w_{a}, w_{b}\right)$, as guaranteed by Eqs. (19)-(21). Accordingly the use of the parameter space $\left(X_{a}, X_{b}, Y\right)$ makes it possible to study the evolution of the universe before doing brute-force numerical calculations.

In the rest of this paper we will only consider the universe with flat extra dimensions, i.e.,

$$
k_{b}=0
$$


for simplicity. In this case the precise value of the extra-dimension size $b$ is not relevant to the evolution of the universe. (We can see it from rescaling the scale factor $b$ in Einstein equations (3)-(5). In the case of $k_{b}=0$, these equations remain the same under the rescaling of $b$.) Accordingly, in the next section we will make use of a two-dimensional parameter space expanded by $X_{a}$ and $Y$ (i.e., the plane of $X_{b}=0$ ) to study the evolution of the universe in various possible eras, from the very early time to the present. (Hereafter we will use $X$ instead of $X_{a}$ for convenience.)

We will discuss three possible eras: the blazing era, the radiation-dominated era, and the matter-dominated era, following the time order. The existence of the blazing era is speculative. In this era the temperature of the universe is so high that particles move relativistically in both ordinary dimensions and extra dimensions (i.e., $T \gg 1 / b$ ) and hence $w_{a}=w_{b}=1 /(3+n)$. In the expanding and cooling process of the universe, after some time the temperature will be lower than the inverse of the size of extra dimensions (i.e., $T<1 / b)$ so that the motion in extra dimensions, i.e., KK modes, cannot be excited and particles are basically at the ground state and therefore pressureless in extra dimensions thereafter. After that time if the temperature is high enough such that dominant particles are still relativistic in ordinary dimensions, we have $w_{a}=1 / 3$ and $w_{b}=0$, as to be designated as "radiation-dominated era" in this paper. Conversely, if the temperature is so low in the later time that the universe is dominated by particles that are nonrelativistic in both ordinary dimensions and extra dimensions, we have $w_{a}=w_{b}=0$, as to be designated as "matter-dominated era".

\section{The evolution in three eras}

\subsection{The blazing era}

In the blazing era, $w_{a}=w_{b}=1 /(3+n)$. In Fig. 1 we plot regions for $\bar{\rho}>0, \bar{\rho}=0$ or $\bar{\rho}<0$, which are obtained from Eq. (19), regions for $\ddot{a}>0, \ddot{a}=0$ or $\ddot{a}<0$ from Eq. (20), flow vectors $d(X, Y) /(u d t)$ from Eqs. (20)-(22) (assuming $u>0$ ), and four trajectories from numerical solutions, where the number of extra dimensions $n$ has been specified to be three for demonstration. The black area denoted by " $\bar{\rho}<0$ " is a forbidden region if the energy density is required to be positive. White dots stand for fixed points where flow vectors are zero both in $X$ and $Y$ directions. The four evolution trajectories correspond to numerical solutions of Eqs. (12) and (13) with the initial conditions $\left(k_{a} / a^{2} u^{2}, v / u\right)=$ (a) $(-0.0625,5)$, (b) $(0.0625,5)$, (c) $(1,-0.9)$, and (d) $(-0.01,-0.1)$ respectively, representing four possible evolution patterns: ${ }^{1}$

(a) acceleration $\rightarrow$ deceleration $\rightarrow$ acceleration, eventually approaching asymptotically the attractor at $(-1,0)$ with stable extra dimensions and zero acceleration, possessing negative spatial curvature.

(b) acceleration $\rightarrow$ deceleration, possessing increasing positive curvature contribution.

\footnotetext{
${ }^{1}$ Note that for all the three eras we will only present some among all the possible evolution patterns because many others are apparently not suitable for describing our universe.
} 


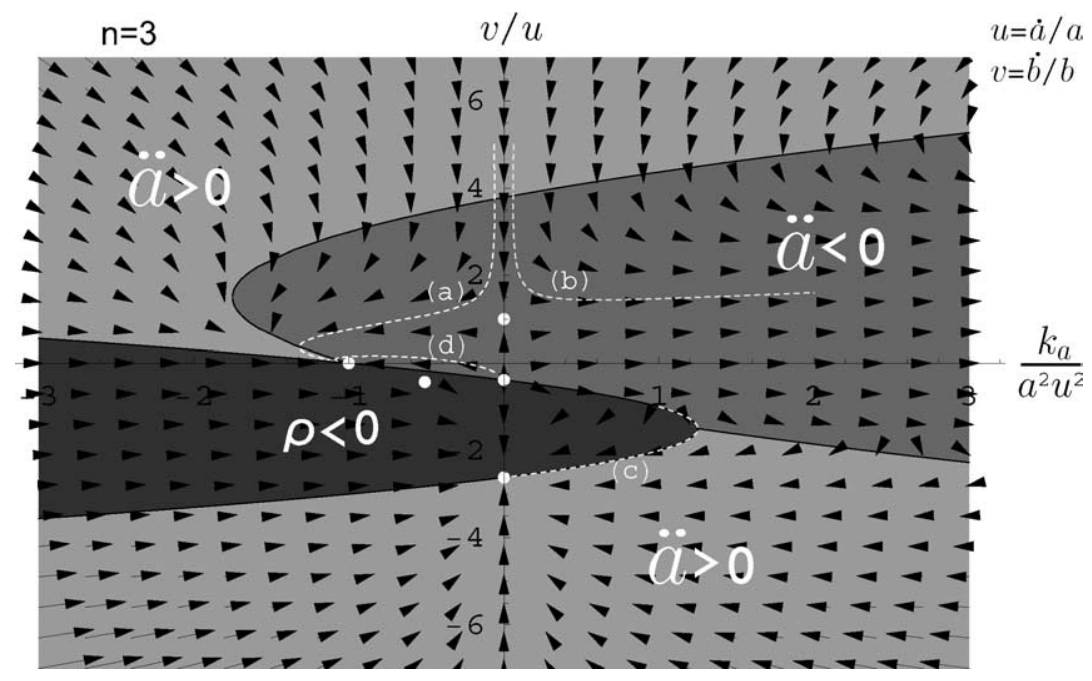

Fig. 1. The $\left(v / u, k_{a} / a^{2} u^{2}\right)$ parameter space describes various states and possible evolution patterns of a blazing universe, where the number of extra dimensions is specified to be three for demonstration. Flow vectors and four evolution trajectories (white dashed curves) are plotted.

(c) deceleration $\rightarrow$ acceleration, eventually approaching asymptotically the attractor at $\{0,-[3+\sqrt{3(n+2) / n}] /(n-1))\}$ with collapsing extra dimensions, possessing decreasing positive curvature contribution in the late time.

(d) deceleration $\rightarrow$ acceleration, eventually approaching asymptotically the attractor at $(-1,0)$ with stable extra dimensions and zero acceleration, possessing negative spatial curvature.

It is essential to ask whether in this model the inflation can take place in this era without introducing the inflaton field. From Fig. 1 we conclude that the universe cannot have a sufficient inflating expansion, which makes the universe nearly flat, in the blazing era unless the extra space is collapsing violently or the expansion rate of the extra space is much larger than that of the ordinary space (i.e., $v \gg u$ ) initially. On the contrary, if the inflation occurs before the blazing era such that the resultant curvature contribution is extremely small, the universe may evolve nearly along the $v / u$-axis during the blazing era. In this case, for a significant part of initial states in the parameter space (more precisely, for $v>[-3+\sqrt{3(n+2) / n}] /(n-1)$ if $k_{a} / a^{2} u^{2} \sim 0$ as required by the inflation) the universe tends to evolve to the state at $\left(k_{a} / a^{2} u^{2}, v / u\right)=(0,1)$, that is, the ordinary space and the extra space tend to synchronize their expansion rates. This feature provides a natural initial condition, $k_{a} / a^{2} u^{2} \sim 0$ and $v / u \simeq 1$, for the succeeding radiation-dominated era.

\subsection{The radiation-dominated era}

In the radiation-dominated era, $w_{a}=1 / 3$ and $w_{b}=0$. Fig. 2 shows regions corresponding to different signs of $\bar{\rho}$ and $\ddot{a}$, flow vectors, five fixed points (denoted by five white dots), 


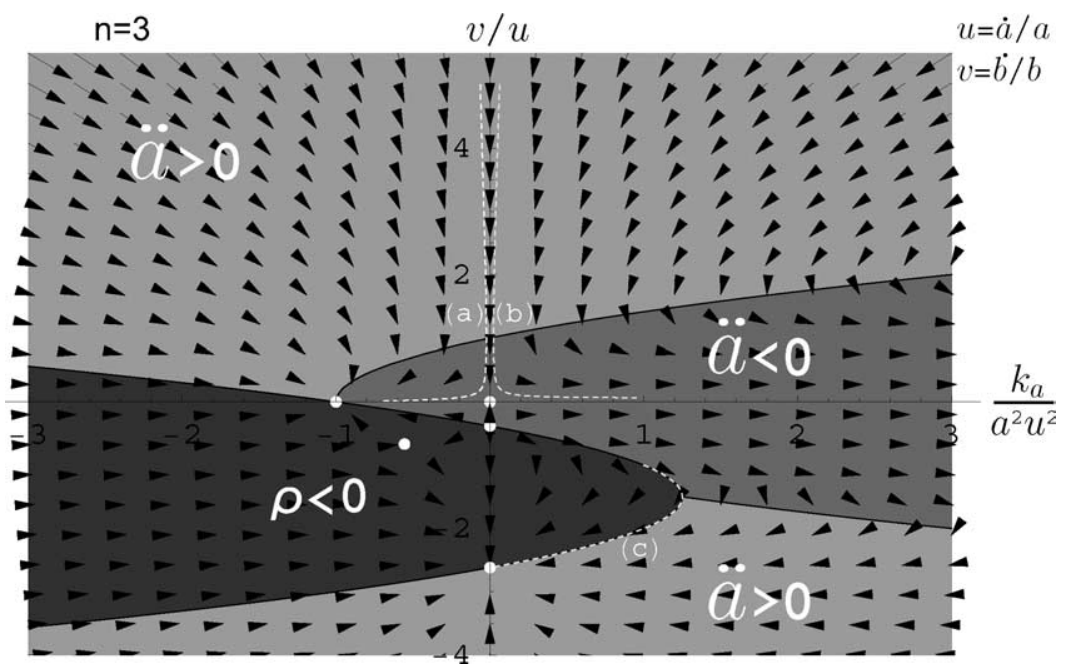

Fig. 2. The $\left(v / u, k_{a} / a^{2} u^{2}\right)$ parameter space describes various states and possible evolution patterns of a radiation-dominated universe, where the number of extra dimensions is specified to be three for demonstration. Flow vectors and three evolution trajectories (white dashed curves) are plotted.

and three evolution trajectories in the parameter space (where $n$ is specified to be three for demonstration). The three trajectories are with respect to initial conditions $\left(k_{a} / a^{2} u^{2}, v / u\right)$ $=(\mathrm{a})(-0.0625,5),(\mathrm{b})(0.0625,5)$, (c) $(1,-0.9)$, representing three possible evolution patterns:

(a) acceleration $\rightarrow$ deceleration, eventually approaching asymptotically the attractor at $(-1,0)$ with stable extra dimensions and zero acceleration, possessing negative spatial curvature.

(b) acceleration $\rightarrow$ deceleration, eventually approaching asymptotically the $k_{a} / a^{2} u^{2}$-axis with stable extra dimensions, possessing increasing positive curvature contribution.

(c) deceleration $\rightarrow$ acceleration, eventually approaching asymptotically the attractor at $\{0,-[3+\sqrt{3(n+2) / n}] /(n-1))\}$ with collapsing extra dimensions, possessing decreasing positive curvature contribution in the late time.

The answer to the question regarding whether the inflation can be generated in this radiation-dominated era without introducing the inflaton field is similar to that in the blazing era. We conclude from Fig. 2 that the universe cannot have a sufficient inflation, which makes the universe flat enough, in this era unless the extra space is collapsing violently or the expansion rate of the extra space is much larger than that of the ordinary space (i.e., $v \gg u$ ) initially.

An important feature in this era is that for about half of initial conditions in the parameter space the corresponding evolution will eventually approach to the states with stable extra dimensions, i.e., $v / u \rightarrow 0$. This implies that the extra dimensions have a significant possibility to be stabilized automatically without resorting to additional artificial mecha- 
nisms. For a negligibly small expansion rate of extra dimensions $v$, we can recover the standard cosmology as follows: With negligible contributions from $v$, zero curvature in the extra space $\left(k_{b}=0\right)$, and equations of state for this era $\left(\bar{p}_{a}=1 / 3 \bar{\rho}\right.$ and $\left.\bar{p}_{b}=0\right)$, Eqs. (6)(8) become

$$
\begin{aligned}
& 3\left[\left(\frac{\dot{a}}{a}\right)^{2}+\frac{k_{a}}{a^{2}}\right]=8 \pi \bar{G} \bar{\rho}, \\
& 2 \frac{\ddot{a}}{a}+\left[\left(\frac{\dot{a}}{a}\right)^{2}+\frac{k_{a}}{a^{2}}\right]=-8 \pi \bar{G} \bar{\rho} / 3, \\
& 3 \frac{\ddot{a}}{a}+3\left[\left(\frac{\dot{a}}{a}\right)^{2}+\frac{k_{a}}{a^{2}}\right]=0,
\end{aligned}
$$

where Eqs. (23) and (24) are exactly the Einstein equations in the standard cosmology and Eq. (25) is a redundant equation that can be derived from the other two equations. (Indeed the fact that one of the above equations is redundant indicates the existence of the solution with $v=0$.) Consequently, with small enough $v$ in this model we can preserve the essential predictions in the standard cosmology, in particular the primordial abundance of light elements produced during big bang nucleosynthesis (BBN), in the radiation-dominated era. In addition, this feature provides a natural initial condition, $k_{a} / a^{2} u^{2} \sim 0$ (guaranteed by inflation presumably) and $v / u \simeq 0$, for the succeeding matter-dominated era.

\subsection{The matter-dominated era}

The evolution in the matter-dominated era, especially the possible accelerating expansion of the present universe, is the main issue that we will use more length to discuss in this paper. In this era the universe is dominated by pressureless matter (without introducing dark energy), therefore $\bar{\rho}=\bar{\rho}_{M}$ and $w_{a}=w_{b}=0$. For demonstration the number of extra dimensions $n$ is chosen to be three for the plots in the parameter space. Fig. 3 shows regions corresponding to various signs of $\bar{\rho}_{(M)}$ and $\ddot{a}$ and the observational bound to the energy density of the matter (mainly the cold dark matter), which is represented by a solid curve denoted by $\Omega_{(M)}=0.35 .^{2}$ Fig. 4 shows flow vectors, five fixed points (denoted by five white dots), and four evolution trajectories in the parameter space. The four trajectories are with respect to initial conditions $\left(k_{a} / a^{2} u^{2}, v / u\right)=(\mathrm{a})(-0.0625,5)$, (b) $(0.0625,5)$, (c) $(1,-0.9),(d)(-0.0625,-0.1575)$, representing four possible evolution patterns (that are somewhat similar to those in the blazing era):

(a) acceleration $\rightarrow$ deceleration $\rightarrow$ acceleration, eventually approaching asymptotically the attractor at $(-1,0)$ with stable extra dimensions and zero acceleration, possessing negative spatial curvature.

(b) acceleration $\rightarrow$ deceleration, possessing increasing positive curvature contribution.

\footnotetext{
2 The observational bound to the energy density of the pressureless matter is $0.15 \lesssim \Omega_{M} \lesssim 0.45$ [22]. In our model, $\Omega_{M} \equiv 8 \pi \bar{G} \bar{\rho}_{(M)} / 3 u_{0}^{2}$, where $u_{0}$ is the present value of the Hubble parameter.
} 


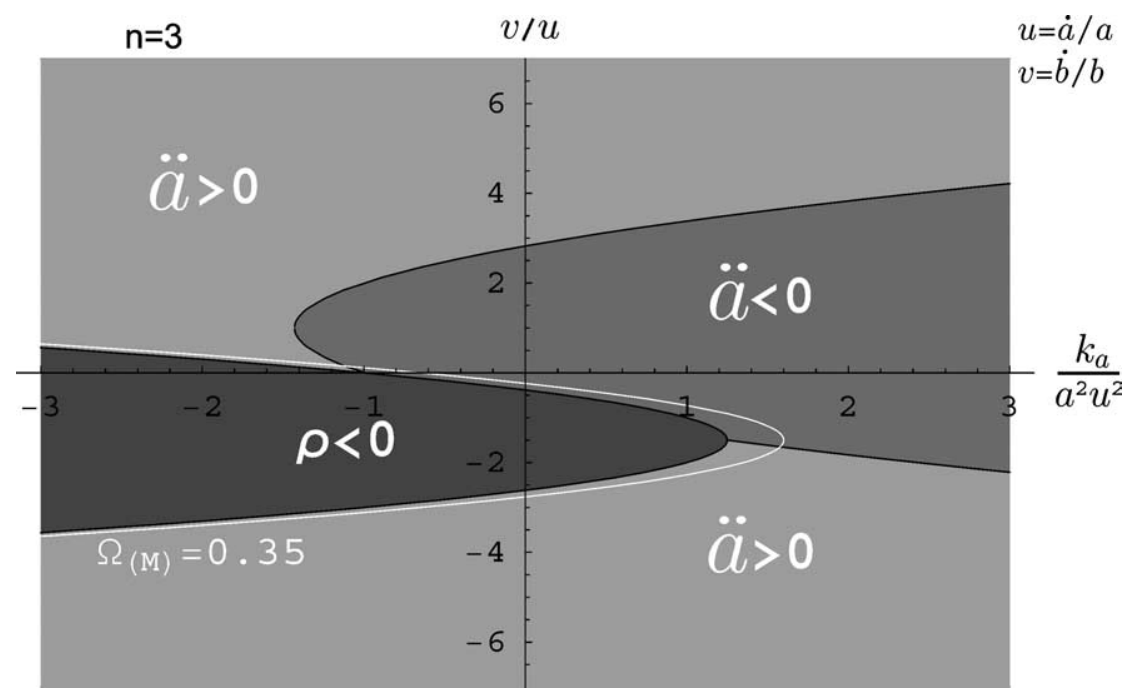

Fig. 3. Various conditions in the $\left(v / u, k_{a} / a^{2} u^{2}\right)$ parameter space for a matter-dominated universe are demonstrated.

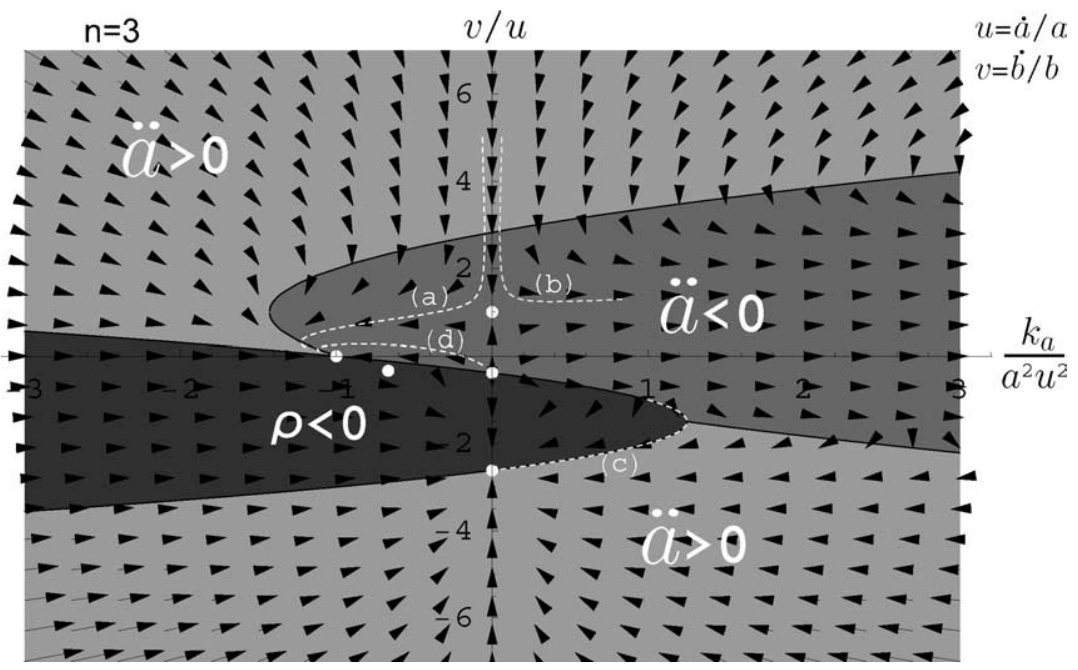

Fig. 4. The $\left(v / u, k_{a} / a^{2} u^{2}\right)$ parameter space describes various states and possible evolution patterns of a matter-dominated universe. Flow vectors and four evolution trajectories (white dashed curves) are plotted.

(c) deceleration $\rightarrow$ acceleration, eventually approaching asymptotically the attractor at $\{0,-[3+\sqrt{3(n+2) / n}] /(n-1))\}$ with collapsing extra dimensions, possessing decreasing positive curvature contribution in the late time.

(d) deceleration $\rightarrow$ acceleration, eventually approaching asymptotically the attractor at $(-1,0)$ with stable extra dimensions and zero acceleration, possessing negative spatial curvature. 
The possible state and the allowed evolution pattern of the universe are constrained by a variety of observations, such as anisotropies of the cosmic microwave background (CMB), large-scale structure (LSS), distance measurements of type Ia supernovae ( $\mathrm{SNe}$ Ia), etc. In addition to the limit to the variation of the Newtonian gravitational constant $G_{N}$ that entails a general difficulty for this scenario [20], we will consider constraints on the "acceleration parameter" $\tilde{q}_{0} \equiv \ddot{a}_{0} / a_{0} u_{0}^{2}$ (i.e., $-q_{0}$ ) and other essential cosmological parameters such as the pressureless matter density parameter $\Omega_{M}$ and the Hubble constant $H_{0}$ (i.e., $u_{0}$ ) (where the subscript ' 0 ' denotes the present time).

As summarized in [21], the bound to $\left|\dot{G}_{N} / G_{N}\right|$ ranges from $10^{-12} \mathrm{yr}^{-1}$ to $10^{-10} \mathrm{yr}^{-1}$ for different observations. In the model with extra dimensions considered here, the Newtonian gravitational constant varies along with the variation of the size of extra dimensions in the way that

$$
G_{N}=\bar{G} / V_{n} \propto b^{-n},
$$

where $V_{n}$ is the volume of the extra space. Accordingly,

$$
\dot{G}_{N} / G_{N}=-n v \text {. }
$$

Consequently, the upper bound to $\left|v_{0}\right|$ is $\left(10^{-12}-10^{-10}\right) \times n^{-1} \mathrm{yr}^{-1}$, and the upper bound to $\left|(v / u)_{0}\right|$ is about $(0.01-1) \times n^{-1} h^{-1}$ (where we have used $u_{0}=100 \mathrm{~h} \mathrm{~km} \mathrm{~s}^{-1} \mathrm{Mpc}^{-1}$ ). (For $h=0.71$ as suggested by WMAP [3] and the special case with $n=3$, the upper bound ranges from 0.0047 to 0.47 .) Therefore the present state of the universe should be around the $k_{a} / a^{2} u^{2}$-axis in the parameter space.

The above constraint on $\left|(v / u)_{0}\right|$, the requirement $\ddot{a}_{0}>0$ from SN Ia data, and $0.15 \lesssim \Omega_{M} \lesssim 0.45$ [22] together imply that the present state of the universe should be in the up-left direction of the fixed point at $(-1,0)$ in the parameter space (Fig. 3$)$, and its evolution should follow type-(a) or type-(d) trajectories in Fig. 4. Type-(d) trajectories are much more favored than type (a) for two reasons: firstly, type-(d) trajectories possess smaller $|v / u|$ through this era so that they are more consistent with the bound to the variation of the Newtonian gravitational constant. Secondly, it has been shown in the previous discussions that in the radiation-dominated era the universe may approach to the states with stable extra dimensions (i.e., $v / u \rightarrow 0$ ), which should be the most probable initial conditions for the succeeding matter-dominated era, accordingly leading to type-(d) trajectories.

There is a boundary between type-(a) and type-(d) trajectories. The intersection of this boundary and the present condition $0.15 \lesssim \Omega_{(M)} \lesssim 0.45$ in the parameter space provides an upper bound, which is about $6.09 \times 10^{-2}$, to the acceleration parameter $\tilde{q}_{0}$. These facts are summarized in Fig. 5, where the point $(-1.173,0.165)$ is the intersection of the curve $\Omega_{(M)}=0.35$ and the boundary between type-(a) and type-(d) trajectories, and the white dashed line denotes one of type-(d) trajectories which pass through the neighborhood of this intersection point. In particular, we note that the maximal-acceleration-parameter state represented by this intersecting point entails small enough $v / u(v / u=0.165)$ so that a moderate bound to the variation of the Newtonian gravitational constant is satisfied. This fact indicates that the essential difficulty from the variation of the Newtonian gravitational constant can be somewhat avoided for type-(d) trajectories. Nevertheless, this value of $\tilde{q}_{0}$ is smaller than that required by SN Ia data together with the results of 


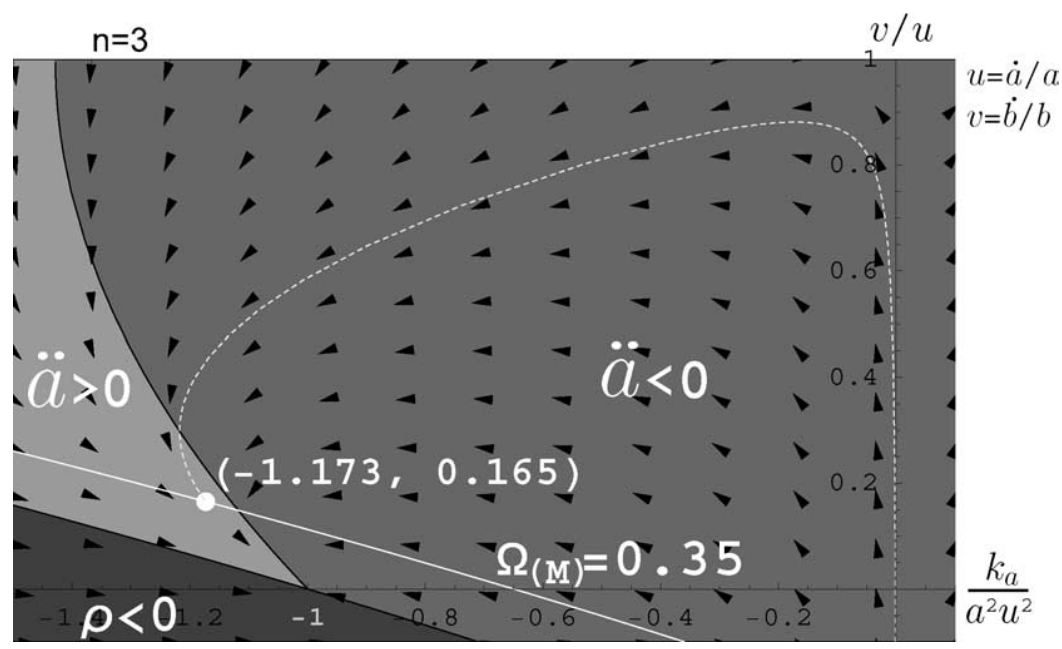

Fig. 5. An enlarged plot for the region where (d)-type trajectories pass. The trajectory (white dashed curve) which entails the largest acceleration parameter $\tilde{q}_{0}$ is plotted.

CMB and LSS observations within the framework of the standard cosmology [23]. According to the result in [23], the most probable values of $\left(\Omega_{M}, w_{X}\right)$ corresponding to the requirement $\tilde{q}_{0}=6.09 \times 10^{-2}$ is about $(0.35,-0.51)$, which is roughly on the margin of the $2.5 \sigma$ confidence interval. (In [23] $w_{X}$ denotes the equation of state of dark energy.)

Now we want to compare the trajectory in Fig. 5, which entails the maximal value of $\tilde{q}_{0}$ among type-(d) trajectories in our model with extra dimensions, to be called "ED model", with that predicted in the standard cosmology in which a flat universe is considered to consist of pressureless matter with the density parameter $\Omega_{M}$ and dark energy with the density parameter $\Omega_{X}\left(=1-\Omega_{M}\right)$ and a constant state parameter $w_{X}\left(w_{X} \equiv p_{X} / \rho_{X}\right)$, to be called "SC model". In the ED model we need to specify the present condition about the essential parameters $\Omega_{(M)}, X_{0}, Y_{0}$, and $u_{0}$ for obtaining an evolution path, i.e., a numerical solution of the Einstein equations (3)-(5), while in the SC model the values of $\Omega_{M}, w_{X}$, and $H_{0}\left(H_{0}=u_{0}\right.$ by definition) are necessary information. In order to make these two evolution paths to be as similar as possible for the present time, we choose the present conditions in the following consistent way: first of all the acceleration parameter $\tilde{q}_{0}$ in both models is specified to be $6.09 \times 10^{-2}$, i.e., the maximal value of $\tilde{q}_{0}$ for type-(d) trajectories in the ED model. As mentioned in the last paragraph about the ED model, the intersection of the curve $\Omega_{(M)}=0.35$ and the (white dashed) trajectory in Fig. 5 is at $(-1.173,0.165)$, which provides the required consistent value of $\left(X_{0}, Y_{0}\right)$. In summary, we choose the following present conditions: $\Omega_{(M)}=0.35, X_{0}=-1.173$ and $Y_{0}=0.165$ for the ED model and $\Omega_{M}=0.35$ and $\Omega_{X}=-0.51$ for the SC model, as well as $H_{0}=u_{0}=71 \mathrm{~km} \mathrm{~s}^{-1} \mathrm{Mpc}^{-1}$ from WMAP [3]. Numerical solutions are shown in Fig. 6. These two evolution paths have similar behavior at the present time but diverge in the earlier time. In particular, the ED path entails a little shorter age. Possible obser- 


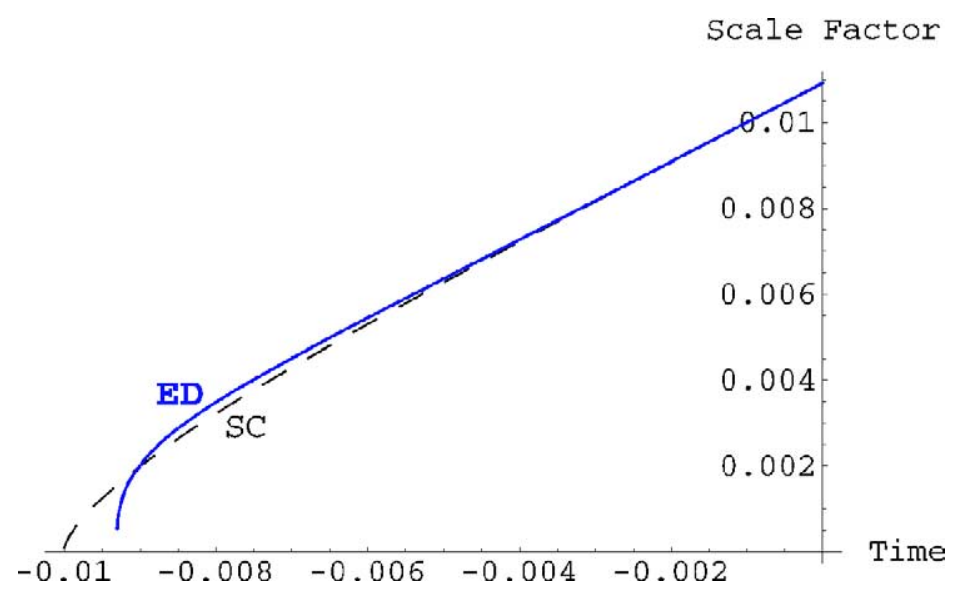

Fig. 6. The evolution of the scale factor $a(t)$ for trajectories in the ED and the SC model.

vational signatures from these discrepancies in the age and the earlier-time behavior are under investigation.

\section{Conclusions and discussions}

We have discussed the evolution of a universe under the influence of flat extra dimensions in various eras via a well-chosen parameter space that provides a systematic, useful, and convenient way for analysis. Since we assume the flatness (zero curvature) of the extra space, the only ingredient of extra dimensions that can affect the evolution of the universe is the evolution of the extra-dimension size (not the precise value of the size), i.e., the expansion rate $v$. We have studied general features of the evolution, thereby exploring whether the picture is viable, subject to constraints from observations, for describing our world.

Through the above studies we have found a natural evolution pattern from the blazing era to the matter-dominated era in this model. In the blazing era, the ordinary space and the extra space tend to synchronize their expansion rates, i.e., $v / u \rightarrow 1$, and meanwhile the universe is decelerating, presuming that an extremely tiny curvature contribution $k_{a} / a^{2} u^{2}$ has been guaranteed initially by inflation. ${ }^{3}$ This tendency provides a natural initial condition, $\left(k_{a} / a^{2} u^{2}, v / u\right)=( \pm \epsilon, 1)$, for the succeeding radiation-dominated era (where ' $\pm \epsilon$ ' indicates a tiny curvature contribution).

In the radiation-dominated era, the expansion rate of the extra space, $v$, tends to approach zero from $v / u \simeq 1$ (as suggested above), meanwhile maintaining the decelerating phase. ${ }^{4}$ It is a good feature for building models with extra dimensions that the expan-

\footnotetext{
3 This tendency corresponds to the fixed point at $\left(k_{a} / a^{2} u^{2}, v / u\right)=(0,1)$ in the parameter space for the blazing era, which is stable in the $Y$ direction but unstable in the $X$ direction.

4 This tendency corresponds to the fixed point at $\left(k_{a} / a^{2} u^{2}, v / u\right)=(0,0)$ in the parameter space for the radiation-dominated era, which is stable in the $Y$ direction but unstable in the $X$ direction.
} 
sion rate of the extra space decreases to zero automatically, that is, extra dimensions are stabilized automatically in this era without resorting to any artificial mechanism. In particular, for small enough $v$ we can recover the standard cosmology (without extra dimensions) and the essential predictions therein (in particular, big bang nucleosynthesis) in the radiation-dominated era. Therefore in our model with extra dimensions the predictions in the standard cosmology in the radiation-dominated era can be preserved in a natural way.

In the matter-dominated era, a natural initial condition, $\left(k_{a} / a^{2} u^{2}, v / u\right)=( \pm \epsilon, 0)$, is provided by the preceding, radiation-dominated era. For the case of a negative curvature, i.e., with the initial condition $(-\epsilon, 0)$, the universe will eventually change its initial decelerating phase to the accelerating one and approach the attractor at $\left(k_{a} / a^{2} u^{2}, v / u\right)=(-1,0)$ with stable extra dimension and significant negative curvature contribution. As we have shown, this evolution pattern is marginally consistent with the limit on the variation of the Newtonian gravitational constant. The maximal acceleration associated with this evolution pattern deviates from that required by SN Ia data together with CMB and LSS observations (within the framework of the standard cosmology) [23] with $2.5 \sigma$ deviation. Thus this evolution pattern is still not ruled out by the present observations.

Although the precise value of the extra-dimension size $b$ provides no direct effect on the evolution of our universe, it is relevant to the existence, the starting and the ending time of these three eras. For example, if the size $b$ is larger than $T_{\mathrm{EQ}}^{-1}$, where $T_{\mathrm{EQ}}$ is the temperature of the universe at the moment of the matter-radiation equality $\left(T_{\mathrm{EQ}} \sim 1 \mathrm{eV}\right)$, there will be no radiation-dominated era. For the same reason, in order to preserve the predictions in big bang nucleosynthesis in the radiation-dominated era when the temperature is around several $\mathrm{MeV}$, the size $b$ at that time needs to be much smaller than $1 \mathrm{MeV}^{-1}$. In addition, because the temperature $T$ of the universe is proportional to $1 / a$ and for the natural evolution pattern we have $0 \leqslant v / u \leqslant 1$ in the radiation-dominated and the matterdominated era, the extra-dimension size $b$ grows slower than $1 / T$ and therefore remains smaller than $1 / T$ in these two eras since the time when $T<1 / b$ (i.e., the beginning of the radiation-dominated era). This provides a consistent check that guarantees the validity of our analysis for the natural evolution pattern in the radiation-dominated and the matterdominated era.

We note that in this natural evolution pattern the expansion rate of extra dimensions will eventually approach zero, consequently extra dimensions being stabilized automatically without resorting to any artificial mechanism, in both the radiation-dominated and the matter-dominated era (for the difficulty in stabilizing extra dimensions in the matterdominated era, see [24]). This is the key ingredient for generating accelerating expansion of the present universe as well as satisfying the limit on the variation of the Newtonian gravitational constant (both caused by the evolution of extra dimensions), meanwhile preserving essential predictions in the standard cosmology for the radiation-dominated era. In particular, we emphasize that the naturalness of this evolution pattern, whose behavior is not sensitive to the initial conditions, indicates a solution to the cosmic coincidence problem ("why now" problem) of dark energy—why dark energy starts dominating the universe now, or, more precisely, why the accelerating phase starts at the present epoch. The existence of this natural evolution pattern implies that the late-time accelerating expansion of 
an open universe ${ }^{5}$ in the matter-dominated era is guaranteed, accordingly the cosmic coincidence problem being solved, in our flat ED model with the help of inflation in the very early time.

Nevertheless, type-(d) trajectories in general entail a too short age of the universe and negative curvature in the ordinary space rather than almost zero curvature suggested by CMB data within the framework of standard cosmology. These features might eventually rule out this evolution pattern for describing our universe. Further detailed studies about the consistency with observations are in progress. Moreover, in order to complete the picture of the scenario for a universe with extra dimensions and find the best-fit trajectory therein, a more general model with nonzero curvature in the extra space is also under investigation.

\section{Acknowledgements}

This work is supported in part through the Taiwan CosPA Project, as funded by the Ministry of Education (MoE 89-N-FA01-1-4), and also supported by the National Science Council, Taiwan, ROC (NSC 92-2112-M-002-051).

\section{References}

[1] Supernova Cosmology Project Collaboration, S. Perlmutter, et al., Astrophys. J. 517 (1999) 565, astro$\mathrm{ph} / 9812133$.

[2] Supernova Search Team Collaboration, A.G. Riess, et al., Astron. J. 116 (1998) 1009, astro-ph/9805201.

[3] C.L. Bennett, et al., Astrophys. J. Suppl. 148 (2003) 1, astro-ph/0302207; D.N. Spergel, et al., Astrophys. J. Suppl. 148 (2003) 175, astro-ph/0302209.

[4] J.L. Sievers, et al., Astrophys. J. 591 (2003) 599, astro-ph/0205387.

[5] ACBAR Collaboration, C.L. Kuo, et al., Astrophys. J. 600 (2004) 32, astro-ph/0212289.

[6] A. Balbi, et al., Astrophys. J. 545 (2000) L1, astro-ph/0005124; Boomerang Collaboration, P. de Bernardis, et al., in: Proceedings of the CAPP2000 Conference, astro$\mathrm{ph} / 0011469$.

[7] L.M. Krauss, M.S. Turner, Gen. Relativ. Gravit. 27 (1995) 1137, astro-ph/9504003; J.P. Ostriker, P.J. Steinhardt, Nature 377 (1995) 600;

A.R. Liddle, D.H. Lyth, P.T. Viana, M. White, Mon. Not. R. Astron. Soc. 282 (1996) 281, astro-ph/9512102.

[8] R.R. Caldwell, R. Dave, P.J. Steinhardt, Phys. Rev. Lett. 80 (1998) 1582, astro-ph/9708069.

[9] J.-A. Gu, W.-Y.P. Hwang, Phys. Lett. B 517 (2001) 1, astro-ph/0105099;

L.A. Boyle, R.R. Caldwell, M. Kamionkowski, Phys. Lett. B 545 (2002) 17, astro-ph/0105318.

[10] J.-A. Gu, W.-Y.P. Hwang, Phys. Rev. D 66 (2002) 024003, astro-ph/0112565.

[11] J.-A. Gu, A way to the dark side of the universe through extra dimensions, in: Proceedings of 2002 International Symposium on Cosmology and Particle Astrophysics (CosPA2002), Taipei, Taiwan, 2002, World Scientific, 2003, pp. 125-133, astro-ph/0209223.

[12] N. Arkani-Hamed, S. Dimopoulos, G. Dvali, Phys. Lett. B 429 (1998) 263, hep-ph/9803315; I. Antoniadis, N. Arkani-Hamed, S. Dimopoulos, G.R. Dvali, Phys. Lett. B 436 (1998) 257, hep-ph/9804398.

[13] I. Antoniadis, Phys. Lett. B 246 (1990) 377.

[14] L. Randall, R. Sundrum, Phys. Rev. Lett. 83 (1999) 3370, hep-ph/9905221; L. Randall, R. Sundrum, Phys. Rev. Lett. 83 (1999) 4690, hep-th/9906064.

5 Actually this open universe is nearly flat in the earlier time. 
[15] T. Kaluza, Sitzungsber. Preuss. Akad. Wiss. Berlin (Math. Phys.) K1 (1921) 966;

O. Klein, Z. Phys. 37 (1926) 895;

O. Klein, Surv. High Energy Phys. 5 (1926) 241.

[16] C. Deffayet, G.R. Dvali, G. Gabadadze, Phys. Rev. D 65 (2002) 044023, astro-ph/0105068.

[17] A. Albrecht, C.P. Burgess, F. Ravndal, C. Skordis, Phys. Rev. D 65 (2002) 123507, astro-ph/0107573.

[18] V. Sahni, Y. Shtanov, JCAP 0311 (2003) 014, astro-ph/0202346.

[19] M.D. Maia, E.M. Monte, J.M.F. Maia, Phys. Lett. B 585 (2004) 11, astro-ph/0208223.

[20] J.M. Cline, J. Vinet, Phys. Rev. D 68 (2003) 025015, hep-ph/0211284.

[21] J.P. Uzan, Rev. Mod. Phys. 75 (2003) 403, hep-ph/0205340.

[22] Particle Data Group Collaboration, K. Hagiwara, et al., Phys. Rev. D 66 (2002) 010001.

[23] S. Perlmutter, M.S. Turner, M.J. White, Phys. Rev. Lett. 83 (1999) 670, astro-ph/9901052.

[24] T. Bringmann, M. Eriksson, M. Gustafsson, Phys. Rev. D 68 (2003) 063516, astro-ph/0303497;

T. Bringmann, M. Eriksson, JCAP 0310 (2003) 006, astro-ph/0308498. 